\title{
Modeling of dispersion and ignition processes of finely dispersed particles of aluminum using a solid propellant gas generator
}

\author{
A. Rychkov ${ }^{1}$, H. Miloshevich ${ }^{2}$, Yu. Shokin ${ }^{2}$, N. Eisenreich ${ }^{3}$ \\ \& V. Weiser ${ }^{3}$ \\ ${ }^{1}$ Institute of Computational Technologies SB RAS, Russia \\ ${ }^{2}$ Prishtin University, Serbia \\ ${ }^{3}$ Fraunhofer Institute of Chemical Technologies, Germany
}

\begin{abstract}
Using numerical modeling, we studied the formation and propagation of a cloud of finely dispersed aluminum particles generated by a special unit under the action of high-temperature combustion products from solid propellant gas generator, as well as the ignition conditions of these particles. We used the Favre-averaged system of Navier-Stokes equations closed by the $q-\omega$ turbulence model to simulate the formation and motion of the cloud of finely dispersed particles. The motion of the polydisperse second phase was described within a stochastic approach that takes into account the effect of the turbulent character of the flow field of the carrier gas on the motion of particles. The finite volume method using the second-order upwind LU difference scheme with TVD-properties is applied for numerically solving this system of equations. The results obtained are in a qualitative agreement with experiments carried out at the Fraunhofer Institute for Chemical Technology (Pfinztal, Germany).

Keywords: mathematical modeling, two-phase flow, processes of ignition and burning, solid propellant gas generators.
\end{abstract}

\section{Introduction}

The scheme of a unit for the fast dispersion of finely dispersed particles is shown in fig. la. It is a cylinder whose central part (a gas generator) is filled with solid monopropellant spherical-form granules with a diameter of a few 
millimeters. The remaining peripheral part of the cylinder contains bulk pulverized finely dispersed aluminum powder that was simulated by a polydisperse medium consisting of spherical particles. There is a metallic net between the gas generator and the bulk material, which does not produce appreciable hydrodynamic resistance to the motion of the gas generator combustion products. The upper and the bottom lids of the cylinder were assumed to be impermeable and fixed so that the particles in the cylinder after the ignition of propellant granules under the effect of gaseous high-temperature high-pressure combustion products moved in a radial direction only. The compaction of particles by the nonstationary action of the combustion products pressure is a rather complex process and an independent problem [1]. Therefore, at the given stage of simulation, the bulk medium between the cylinder heads was supposed to move in the regime of 'plug' pneumatic transport with the maximum permissible level of porosity for spherical particles equal to 0.42 [1]. After the left boundary of the 'plug' left the cylinder, the process of its destruction and the formation of a cloud of particles under the effect of outflowing high-temperature combustion products started, as well as their ignition and combustion.

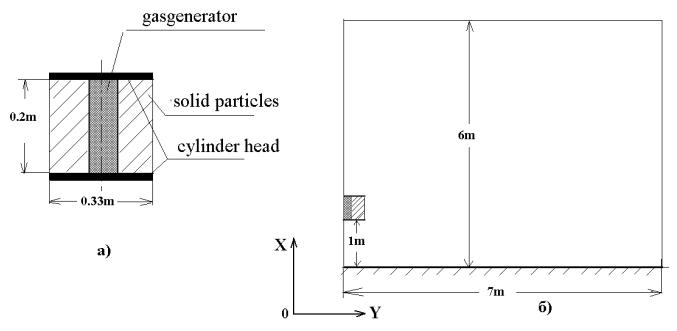

Figure 1: $\quad$ The scheme of the unit.

\section{Description of model and basic equations}

The pressure of the combustion products of propellant granules at the moment the bulk material plug leaves the cylinder is $10-15 \mathrm{MPa}$, the efflux into the ambient medium is supersonic and the turbulent character of the flow is to be taken into account. Therefore, in this paper, we used the Favre-averaged system of Navier-Stokes equations closed by the $q-\omega$ turbulence model to simulate the formation and motion of the cloud of finely dispersed particles [2]. The motion of the polydisperse second phase was described within a stochastic approach [3] that takes into account the effect of the turbulent character of the flow field of the carrying gas on the motion of particles.

In describing the mathematical model of the processes studied, we adopted the following assumptions.

- The flow is turbulent, two-phase, axisymmetric, and nonstationary. The outflow occurs into the static atmosphere of the standard composition. 
The gas generator combustion products represent a nonreacting mixture of an inert component and an oxidizer whose oxidation potential was simulated by some weight fraction of oxygen $\beta$.

- The second phase consists of polydisperse aluminium spherical particles and comprises $\mathrm{N}$ fractions, inside each of them the size of the particles is identical. The particle collisions at the given stage of simulation are disregarded.

- The temperature distribution over a particle volume is assumed to be homogeneous.

- The gas output from solid propellant granules was simulated by the source terms in the mass and energy conservation equations.

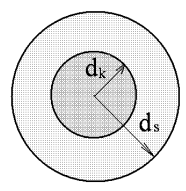

Figure 2: The particle combustion model.

In order to describe the combustion of aluminum particles, we use the model of a contractile metallic core of diameter $d_{i, k}$ and an oxide shell of diameter $d_{i, s}$. The scheme of this model is shown in fig. 2.

The combustion of the $\mathrm{i}$-th particle is supposed to be described by the onestage overall reaction $\mathrm{Al}+3 / 4 \mathrm{O}_{2}=1 / 2 \mathrm{Al}_{2} \mathrm{O}_{3}$, its linear velocity is determined by the formula in [4].

$$
\frac{d\left(d_{i, k}\right)}{d t}=-\frac{10^{-3} \cdot Y_{O_{2}}\left(p \cdot 10^{-5}\right)^{0.1} T^{0.2}}{0.00735 \cdot\left(d_{i o} \cdot 10^{6}\right)^{0.8}}
$$

where $d_{i 0}$ is the initial particle diameter; $Y_{\mathrm{O}_{2}}$ is the mass fraction of the oxidizer; $p, T$ are the pressure and the temperature. The decrease in the mass of a particle nucleus in combustion is described by the dependence

$$
\frac{d m_{i}^{(A l)}}{d t}=\rho_{A l} d_{i, k}^{2} \frac{\pi}{2} \frac{d\left(d_{i, k}\right)}{d t}
$$

The oxide shell mass, on the one hand, increases due to the formation of the oxide in the combustion process and, on the other hand, may decrease due to the escape of $\mathrm{Al}_{2} \mathrm{O}_{3}$ from the shell surface in the form of submicron particles. Therefore, the change in the mass of the oxide shell was written as 


$$
\frac{d m_{i}^{\left({ }^{\left(A l_{2} \mathrm{O}_{3}\right)}\right.}}{d t}=-\frac{d m_{i}^{(A l)}}{d t}\left(1+\frac{3}{4} \frac{M_{O_{2}}}{M_{A l}}-\alpha\right)
$$

where $\alpha$ is an empirical coefficient taking into account the process of the oxide escape from the shell surface. The submicron particles are assumed to be in a local equilibrium with the carrying gas. Therefore, their mass is added to the inert component of the gas generator combustion products and their energy to the total energy of the carrying gas. Further, an aluminum particle is supposed to start burning when the particle temperature reaches a certain value, i.e., the ignition temperature $T_{i g}$.

The system of equations describing this flow has the form:

$$
\begin{gathered}
\frac{\partial \rho}{\partial t}+\frac{\partial}{\partial x}(\rho u)+\frac{1}{r} \frac{\partial}{\partial r}(r \rho v)=M_{g e n}+\left(\frac{3}{4} \frac{M_{O_{2}}}{M_{A l}}-\alpha\right) \sum_{i=1}^{N}<\frac{d m_{i}^{A l}}{d t}>n_{i} \\
\frac{\partial \rho Y_{O_{2}}}{\partial t}+\frac{\partial}{\partial x}\left(\rho u Y_{O_{2}}+q_{x, O_{2}}\right)+\frac{1}{r} \frac{\partial}{\partial r} r\left(\rho v Y_{O_{2}}+q_{r, O_{2}}\right)= \\
\frac{3 M_{O_{2}}}{4 M_{A l}} \sum_{i=1}^{N}<\frac{d m_{i}^{A l}}{d t}>n_{i}+\beta \cdot M_{g e n} \\
\frac{\partial \rho Y_{N_{2}}}{\partial t}+\frac{\partial}{\partial x}\left(\rho u Y_{N_{2}}+q_{x, N_{2}}\right)+\frac{1}{r} \frac{\partial}{\partial r} r\left(\rho v Y_{N_{2}}+q_{r, N_{2}}\right)=0 \\
\frac{\partial \rho Y_{M}}{\partial t}+\frac{\partial}{\partial x}\left(\rho u Y_{M}+q_{x, M}\right)+\frac{1}{r} \frac{\partial}{\partial r} r\left(\rho v Y_{M}+q_{r, M}\right)= \\
-\alpha \sum_{i=1}^{N}<\frac{d m_{i}^{A l}}{d t}>n_{i}+(1-\beta) M_{g e n} \\
\left.<\frac{d m_{i}^{A l}}{d t}>Q_{p}\right]+Q_{g e n} M_{g e n} \\
\frac{\partial \rho u}{\partial t}+\frac{\partial}{\partial x}\left(\rho u_{r r}^{2}+p+\tau_{x x}\right)+\frac{1}{r} \frac{\partial}{\partial r} r\left(\rho u v+\tau_{x r}\right)=-\sum_{i=1}^{N} n_{i}<m_{p, i} \frac{d u_{i}}{d t}> \\
\frac{\partial \rho v}{\partial t}+\frac{\partial}{\partial x}\left(\rho v u+\tau_{r x}\right)+\frac{1}{r} \frac{\partial}{\partial r} r\left(\rho v^{2}+p+\tau_{r r}\right)=p-\sum_{i=1}^{N} n_{i}<m_{p, i} \frac{d v_{i}}{d t}> \\
\frac{\partial \rho}{\partial x}\left(u(\rho E+p)+u \tau_{x x}+v \tau_{x r}+\varphi_{x}\right)+\frac{d}{r} \frac{\partial}{\partial r} r\left(v(\rho E+p)+u \tau_{x r}+\right. \\
d t
\end{gathered}
$$




$$
\begin{gathered}
\frac{\partial \rho q}{\partial t}+\frac{\partial}{\partial x}\left(\rho u q+\tau_{x, q}\right)+\frac{1}{r} \frac{\partial}{\partial r} r\left(\rho u q+\tau_{r . q}\right)=S_{q} \\
\frac{\partial \rho \omega}{\partial t}+\frac{\partial}{\partial x}\left(\rho u \omega+\tau_{x, \omega}\right)+\frac{1}{r} \frac{\partial}{\partial r} r\left(\rho u \omega+\tau_{r . \omega}\right)=S_{\omega} \\
p=\rho T R_{0}\left(\frac{Y_{O_{2}}}{M_{O_{2}}}+\frac{Y_{N_{2}}}{M_{N_{2}}}+\frac{Y_{M}}{M_{M}}\right)
\end{gathered}
$$

The equations of the motion of the $i$-th particle along its path are written as

$$
\begin{gathered}
\frac{d m_{i}^{A l}}{d t}=-\rho_{A l} d_{i, k}^{2} \frac{\pi}{2} \cdot N_{i} \frac{10^{-3} \cdot Y_{O_{2}}\left(P \cdot 10^{-5}\right)^{0.1} T^{0.2}}{A \cdot\left(d_{i o} \cdot 10^{6}\right)^{n-1}} \\
\frac{d m_{i}^{A l_{2} O_{3}}}{d t}=-\left(\frac{M_{A l_{2} O_{3}}}{2 \cdot M_{A l}}-\alpha\right) \frac{d m_{i}^{A l}}{d t} \\
\frac{d u_{i}}{d t}=\frac{3}{4} \frac{\mu C_{D i} \operatorname{Re}_{p . i}}{\rho_{i, p} d_{i, s}^{2}}\left(u+u^{\prime}-u_{i}\right) \\
\frac{d v_{i}}{d t}=\frac{3}{4} \frac{\mu C_{D i} \operatorname{Re}_{p, i}}{\rho_{i, p} d_{i, s}^{2}}\left(v+v^{\prime}-v_{i}\right) \\
\frac{d T_{i}}{d t}=\frac{6}{C_{i}} \frac{\lambda \cdot N u}{\rho_{p, i} d_{i, s}^{2}}\left(T-T_{i}\right) \\
\frac{d x_{i}}{d t}=u_{i} \\
\frac{d y_{i}}{d t}=v_{i}
\end{gathered}
$$

where $q=\sqrt{k}, \omega=\varepsilon / k$ are 'turbulent' variables related to the turbulent kinetic energy $\mathrm{k}$ and its dissipation rate $\varepsilon ; E=C_{v} T+\left(u^{2}+v^{2}\right) / 2$ is the total specific energy; $H=E+p / \rho$ is the specific enthalpy; $\mu$ and $\mu_{\mathrm{t}}$ are the molecular and turbulent viscosity, respectively; $p, T$ are the static pressure and temperature; $\mathrm{R}$ is the gas constant; $Y_{O_{2}}, Y_{N_{2}}, Y_{M}, M_{O_{2}}, M_{N_{2}}$ and $\mathrm{M}_{\mathrm{M}}$ are the mass fractions and molecular weights of oxygen, nitrogen, and the inert component, respectively. The values $M_{g e n}$ and $Q_{g e n}$ are the mass and energy sources simulating the inflow of high-temperature combustion products from the gas generator.

$$
\begin{gathered}
S_{q}=C_{q 1}\left(C_{\mu} S / \omega^{2}-2 D /(3 \omega)-1\right) \rho \omega q, \\
S_{\omega}=\left[C_{\omega 1}\left(C_{\mu} S / \omega^{2}-C_{\omega 3} D / \omega\right)-C_{\omega 2}\right] \rho \omega^{2}
\end{gathered}
$$


The constants in the description of the turbulence model have the following values: $C_{\omega 1}=0.55, C_{\omega 2}=0.833, C_{\omega 3}=0.666, C_{\mu}=0.09, C_{q 1}=0.5$,

$$
\begin{gathered}
\mu_{t}=C_{\mu} \rho q^{2} / \omega, \tau_{r x}=\tau_{x r}=\mu_{e}\left(\frac{1}{r} \frac{\partial r u}{\partial r}+\frac{\partial v}{\partial x}\right), \phi_{x}=-\lambda_{e} \frac{\partial T}{\partial x} \\
\phi_{r}=-\lambda_{e} \frac{\partial T}{\partial r}, \mu_{e}=\mu+\mu_{t}, \lambda_{e}=\lambda+\lambda_{t}, \lambda_{t}=\mu_{t} C_{p} / \operatorname{Pr}_{t}, D=\frac{\partial u}{\partial x}+\frac{1}{r} \frac{\partial r v}{\partial r} \\
S=\left(u_{i, j}+u_{j, i}\right) u_{i, j}-2 / 3 u_{k, k}^{2}, q_{x, k}=-\left(\rho D_{k}+\frac{\mu_{t}}{S c_{t}}\right) \frac{\partial Y_{k}}{\partial x} \\
q_{r, k}=-\left(\rho D_{k}+\frac{\mu_{t}}{S c_{t}}\right) \frac{\partial Y_{k}}{\partial r}, \mathrm{k}=O_{2}, N_{2}, M
\end{gathered}
$$

and $\operatorname{Pr}_{t}$ is the turbulent Prandtl number.

The terms in the angular brackets indicate averaging over the volume of the cell in a difference grid; $n_{i}$ is the concentration of particles of the $i$-th fraction in the cell at the given instant

$$
C_{D i}=\left\{\begin{array}{l}
\frac{24}{\operatorname{Re}_{p i}}\left(1+\frac{\operatorname{Re}_{p i}^{2 / 3}}{6}\right) \\
0.44, \quad \text { if } \operatorname{Re}_{p i}>10^{3}
\end{array}, \text { if } \operatorname{Re}_{p i} \leq 10^{3}, \operatorname{Re}_{p i}=\frac{d_{i, s} \rho\left|\vec{V}-\vec{V}_{i}\right|}{\mu}\right.
$$

where $u^{\prime}, v^{\prime}$ are the random vector components of the disturbed gas velocity $\vec{v}^{\prime}=\left(u^{\prime}, v^{\prime}\right) ; \vec{v}^{\prime}=\sqrt{2 k / 3} \operatorname{erfc}^{-1}\left(N_{r}\right) \vec{V} /|\vec{V}|, \vec{V}$ is the averaged velocity vector; $N_{r}$ is a random number from the range $[-1,+1] ; \operatorname{erfc}^{-1}()$ is the inverse error function; $\quad C_{i}$ is the specific heat of the $i$-th particle, $x_{i}$ and $y_{i}$ are its coordinates.

The diameter of the aluminium oxide shell $d_{i, s}$ of each $i$-th particle was determined from the equality

$$
m_{i}^{A l}+m_{i}^{A l_{2} O_{3}}=\frac{\pi}{6}\left[d_{i, k}^{3} \cdot \rho_{A l}+\left(d_{i, s}^{3}-d_{i, k}^{3}\right) \rho_{A l_{2} O_{3}}\right]
$$

The mean particle density is

$$
\rho_{i, p}=\frac{6\left(m_{i}^{A l}+m_{i}^{A l_{2} O_{3}}\right)}{\pi \cdot d_{i, s}^{3}}
$$

The domain of the solution to the system of eqns (4) - (20) and its size in meters are shown in fig. $1 \mathrm{~b}$, where the OY-axis is horizontally directed, the OX-axis is 
vertically directed. The domain was bounded from below either by an underlying surface (pulverization near the Earth surface) or was not bounded at all (pulverization in an infinite space). The boundary conditions for the carrying gas were given as follows. The flow symmetry condition was given along the OXaxis, the impermeability condition on the surfaces of the cylinder lids and on the underlying surface, and the nonreflective boundary condition on the other boundaries. The inelastic reflection condition was given for the particles on the underlying surface

$$
V_{p i, n}^{-}=-\left(0.993-1.76 \Theta+1.56 \Theta^{2}-0.49 \Theta^{3}\right) V_{p i, n}^{+}, V_{p i, \tau}^{-}=\frac{5}{7} V_{p i, \tau}^{+}
$$

where $\vec{V}_{p i, n}^{+}, \vec{V}_{p i, n}^{-}, \vec{V}_{p i, \tau}^{+}, \vec{V}_{p i, \tau}^{-}$are the normal and tangential velocities of the $i$-th particle before $(+)$ its drop and after $(-)$ its reflection; $\Theta$ is the angle of incidence. The initial condition for the gas and the particles was a stationary state. The mass increase $M_{\text {gen }}$ of the gas generator combustion products in eqns (5), (7), (10) was determined from the solution of the equation

$$
\frac{d M_{g e n}}{d t}=-\rho_{g r} S \cdot r_{b g} N_{g g}^{0}
$$

where $\rho_{g r}, S$ and $r_{b g}$ are the density of a propellant granule, its current surface and the combustion rate, respectively; $N_{g g}^{0}$ is the number of granules in the unit volume of a solid propellant charge, which remains constant in combustion and is determined from the initial conditions.

\section{Numerical method}

The finite volume method using the second-order upwind LU difference scheme with TVD-properties [8] is applied for solving numerically the system (4) (13) for carrier gas. The scheme is close to the scheme from [5]. As well know the stiffness of this system is main difficult by numerical solution of it at low Max numbers. To circumvent this problem we used the preconditioned matrix much as in [5]. We calculated the particle motion by the A-stable difference scheme of the second-order of accuracy [6]. The iterative process is organized to take into account effect of the second phase on movement of carrier gas.

\section{Some computational results}

The calculations were carried out for the following initial conditions: the mass of finely dispersed aluminum particles is $2 \mathrm{~kg}$; the solid propellant charge of the gas generator is $0.2 \mathrm{~kg}$; the combustion rate of granules $r_{b g}=5\left(p / p_{0}\right)^{0.6}[\mathrm{~mm} / \mathrm{s}]$; 
the heat output of the propellant $Q_{\text {gen }}=1780[\mathrm{KJ} / \mathrm{kg}]$. The number of the fractions of particles was five, the ignition temperature of an aluminium particle $T_{i g}=1300 \mathrm{~K}$. Fig. 3 shows the time variations in the total heat generation of burning aluminium particles $\mathrm{Q}[\mathrm{J}]$.

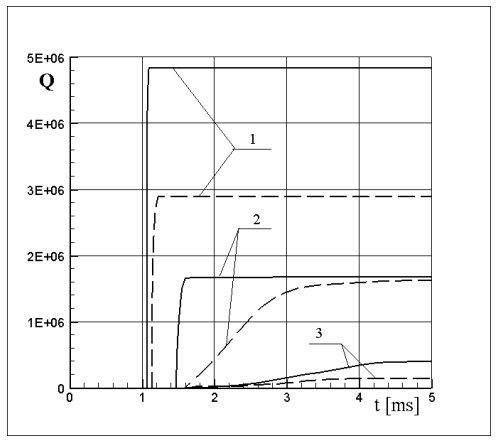

Figure 3: The heat generation of burning particles.

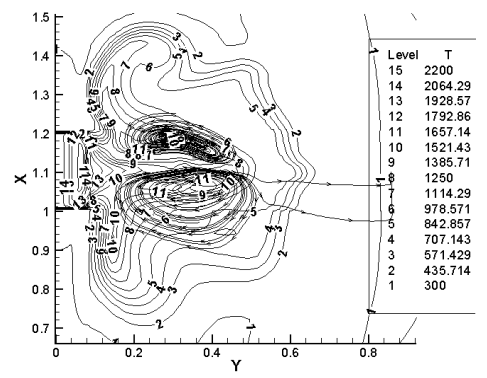

Figure 5: Flow field isotherms for particles with $d_{43}=1.3 \mu \mathrm{m}$.

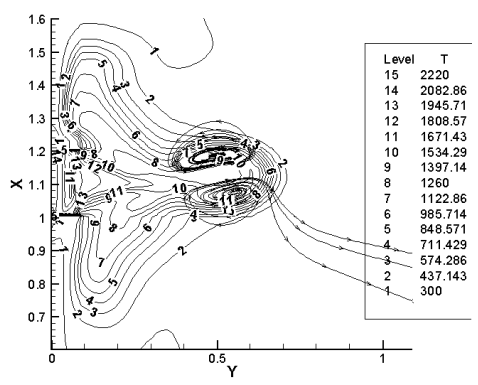

Figure 4: Flow field isotherms for particles with $d_{43}=0.8 \mu \mathrm{m}$.

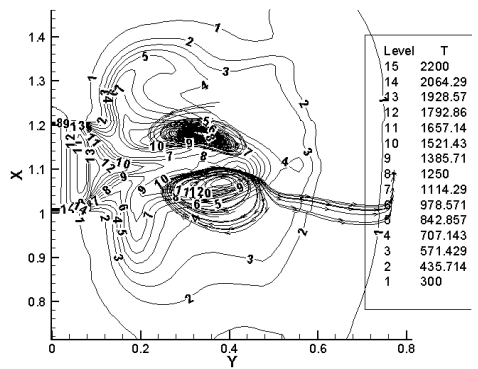

Figure 6: Flow field isotherms for particles with $d_{43}=28 \mu \mathrm{m}$.

The digits refer to the mean mass sizes of particles of $d_{43}=0.8 \mu \mathrm{m}, 1.3 \mu \mathrm{m}$, and $28 \mu \mathrm{m}$, respectively. The solid lines indicate the computational results in the presence of an oxidizer in the gas generator combustion products $(\beta=0.2)$, the dotted lines give results in its absence $(\beta=0)$. As can be seen, the presence of an oxidizer contributes to an earlier ignition of aluminium particles and increases the completeness of its combustion. For the particles with $d_{43}=0.8 \mu \mathrm{m}$ the completeness of combustion amounted to $24.5 \%$ and $9.2 \%$, for $d_{43}=1.3 \mu \mathrm{m}$ to 
$2.9 \%$ and $2.3 \%$, and for $d_{43}=28 \mu \mathrm{m}$ to $0.25 \%$ and $0.23 \%$, respectively. The calculations showed that the structure of the flow, with a forming particle cloud streamlined by the carrying gas, is rather complex and to a large degree specified by the interphase reaction intensity and the heat generation of burning particles. Figs. 4-6 depict the flow field isotherms at time $t=5 \mathrm{~ms}$ for the above three mean mass sizes of particles. The positions of instant paths of gas particles, which give an idea of the eddy structure of the flow are also shown.

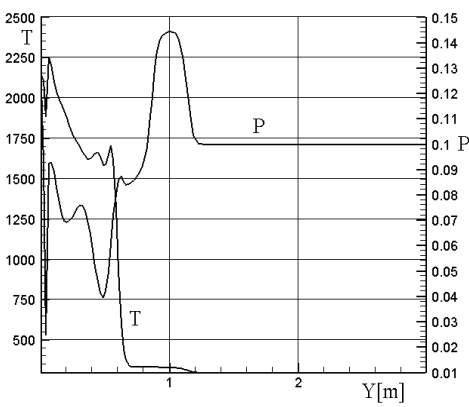

Figure 7: Parameters for particles with $\mathrm{d}_{43}=0.8 \mu \mathrm{m}$.

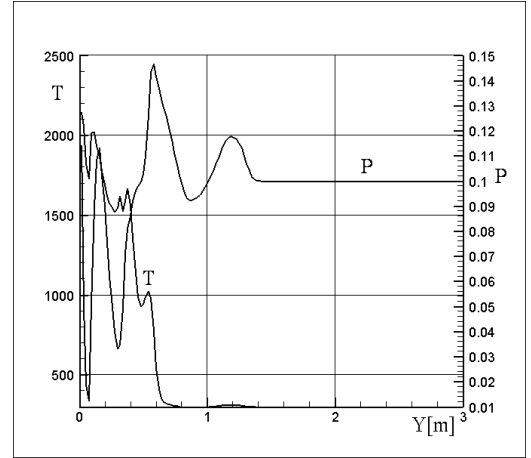

Figure 8: Parameters for particles with $d_{43}=1.3 \mu \mathrm{m}$.

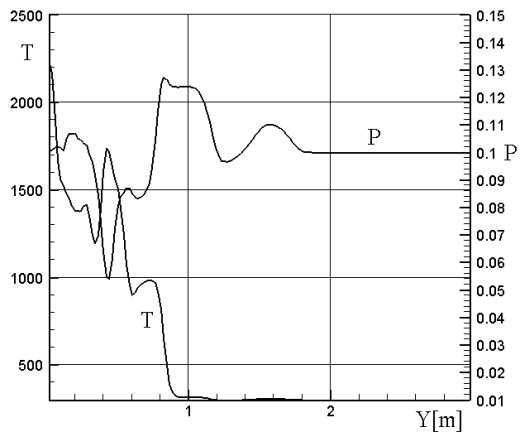

Figure 9: $\quad$ Parameters for particles with $d_{43}=28 \mu \mathrm{m}$.

As can be seen, for small-sized particles with rather high completeness of combustion (fig. 4) the temperature of the front part of an outflowing jet is rather high. At the same time in the case of coarse particles, the 'breakdown' of the jet occurs faster and its penetration into the environment is less intense. Figures 7-9 give the distributions of temperature $\mathrm{T}[\mathrm{K}]$ and pressure $\mathrm{P}[\mathrm{MPa}]$ along the OY-axis for $\mathrm{x}=1.1 \mathrm{~m}$ for the same versions of the flow, which can be used to judge the wave pattern of the outflow process. 
For small-sized particles (see figs. 7 and 8), we observe a strong increase in pressure behind the rarefaction wave due to the strong retardation of the gas by the particles and its subsequent intense oscillations, which induces reverse eddy flows.

The maximum pressure peak even at a distance of one meter remains relatively safe for a human organism. In the case of coarse particles, whose force interaction with the carrying gas is weak, the pressure peak is lower.

The computational results were compared with physical experiments carried out for a segment (a quarter of the cylinder) of this unit. Quantitative comparisons are impossible in view of the lateral interaction of the outflowing jet with the environment in this experiment. However, as to the dynamics of the formation and propagation of the particle cloud, the qualitative agreement proved sufficiently satisfactory [7].

\section{References}

[1] Dullien, F. A. Porous Media Transport and Pore Structure. Academic Press, New York, 1979.

[2] Coakley, T. J. Turbulence modeling for high speed flows. AIAA Paper 920436, 1992.

[3] Crow, C. T. Review - Numerical models for dilute gas-particles flows. Trans. ASME J. Fluid Engrg., 104, pp. 297-303, 1982.

[4] Beckstead, M.W. Correlating aluminum burning times. Combustion, Explosion, and ShockWaves, 41(5), pp. 533-546, 2005.

[5] Chen, K. and Shuen Ji. Three-dimensional coupled implicit method for spray combustion flows at all speed. AIAA Paper 94-307, 1994.

[6] Rychkov, A. D. Mathematical Modelling of Gasdynamic Processes in Channels and Nozzels. Novosibirsk, Nauka, 1988 (in Russian).

[7] Rychkov, A. Schneider, H. Shokina, N. and Eisenreich, N. Numerical and experimental investigation of the gas-dynamic aspect in the dispersal process of $\mu$-sized energetic particles. Proc. of the 33rd International Annual conference of ICT 'Energetic Materials-Synthesis, Production and Application', Karlsruhe, Germany, pp. 140.1-140.12, 2002.

[8] Yoon, S. and Jameson, A.. An LU-SSOR scheme for the Euler and Navier-Stokes equations. AIAA Paper, 87-600, 1987. 